\title{
HOPX Crossover Operator for the Fixed Charge Logistic Model with Priority Based Encoding
}

\author{
Ahmed Lahjouji EI Idrissi ${ }^{1}$, Chakir Tajani ${ }^{2}$ Mohamed Sabbane ${ }^{3}$ \\ ${ }^{1,3}$ Faculty of Science Meknes, Moulay Ismail University, Morocco \\ ${ }^{2}$ Polydisciplinary faculty of Larache, AbdelmalekEssaadi University, Morocco
}

\begin{abstract}
Article Info
Article history:

Received Mar 10, 2018

Revised Jul 25, 2018

Accepted Aug 10, 2018

\section{Keyword:}

Genetic algorithm

Logistic model

Priority based encoding

Transportation problem

ABSTRACT

In this paper, we are interested to an important Logistic problem modelised us optimization problem. It is the fixed charge transportation problem (FCTP) where the aim is to find the optimal solution which minimizes the objective function containig two costs, variable costs proportional to the amount shipped and fixed cost regardless of the quantity transported. To solve this kind of problem, metaheuristics and evolutionary methods should be applied. Genetic algorithms (GAs) seem to be one of such hopeful approaches which is based both on probability operators (Crossover and mutation) responsible for widen the solution space. The different characteristics of those operators influence on the performance and the quality of the genetic algorithm. In order to improve the performance of the GA to solve the FCTP, we propose a new adapted crossover operator called HOPX with the priority-based encoding by hybridizing the characteristics of the two most performent operators, the Order Crossover (OX) and Positionbased crossover (PX). Numerical results are presented and discussed for several instances showing the performance of the developed approach to obtain optimal solution in reduced time in comparison to GAs with other crossover operators.
\end{abstract}

Copyright $\odot$ 2018Institute of Advanced Engineering and Science. All rights reserved.

\section{Corresponding Author:}

Ahmed Lahjouji El Idrissi,

Departement of Mathematics,

Faculty of Science Meknes, Morocco.

Email: idrissila@gmail.com

\section{INTRODUCTION}

Logistic model are the most important problem that need to be optimized for the smooth operation of the entire supply chain [1]. It determines the number and type of plants, warehouses and distribution centers (DCs) to be used. It also establishes distribution channels and the quantity of products to be shipped from suppliers for each customer. Logistic model covers a wide range of formulations from linear deterministic models to nonlinear stochastic complexes ones.

For the first formulation called linear logistic model problem or liner transportation problem. It is a network optimization problem introduced by Hitchcock [2] which consists to minimize the total cost in order to transport homogeneous products from several sources to several deposits satisfying the limits of supply and demand. This model can be find in industry, planning, communication network, scheduling, transportation and attribution. Several search attacks the linear logistics models which can be solved by the simplex method introduced by George Dantzig in 1947 [3]. Also, it can be solved by approximation methods such us the method of Russell and the method of Vogel [4], [5].

The second formulation, which is the objective of this study, is the fixed charge logistic model. The literature around this first case extension is very rich. Hirsch and Danzig in [6] was the first to formulate the FCTP us extension of the liner logistic model. Many practical transportation and distribution problems, such as the minimum cost network flow (transshipment) problem with a fixed-charge for logistics, can be 
formulated as fixed-charge logistic model. For instance, a fixed cost may be incurred for each shipment between a given plant and a given customer and a facility of a plant may result in a fixed amount on investment. The FCTP takes these fixedcharge into account, so that the TP can be considered as an FCTP with equal fixed costs of zero for all routs.

Balainski in 1961 modified the FCTP to a linear integer problem [7]; he observed that there is an optimal solution for the modified version of FCTP. Adlakha in [8] proposed a method which consists of two parts; it gets the best initial solution in the first part and uses techniques to improve this solution and to check its optimality.

The genetic algorithm (GA) is an evolutionary algorithm that represents a famous metaheuristic proposed by Jhon Holland in 1975 [9] which is inspired by biological mechanisms such as Mendel's laws and the theory of evolution proposed by Charles Darwin. It uses the same vocabulary as in biology and classical genetics; so we speak of gene, chromosome, population [10]-[13].

The genetic algorithm has been used to solve many combinatorial problems including FCTP [14]. Its main advantage is that it allows a good combination between the exploitation of solutions and the exploration of the research space. This is established as a function of the GA parameters respectively. However, its disadvantage lies in two points; a computational time large enough to be able to converge towards the optimal solution and the convergence that is a big problem for GAs. In addition, the different characteristics of the genetic operators influence on the performance and the quality of the GA. For this reason and in order to improve the performance of the GA to solve the FCTP, we propose a new adapted crossover operator called HOPX with the priority-based encoding by hybridizing the characteristic of the two most performent operators, the Order Crossover (OX) and Position-based crossover (PX).

This paper is organized as follows: The second section gives an overview of the formulation of the fixed charge logistic model. The third part presents the GA as a method of resolution and these parameters which have influenced the quality of the results. In addition to the proposed hybrid crossover operator called HOPX. The fourth section deals with the development and implementation of the GA with the new operator where several numerical results are presented showing his performance in comparison with variants operators.

\section{LOGISTICS MODEL DESCRIPTION AND FORMULATION}

Logistic is a profound factor on the added value of each business. Examines each of this activity is done at several levels: logistic engineering, technical publications, procurement... Suggests to each element a separate responsibility towards the product arrived at the customer or the consumer in the complex network [15].

Considering the following graph $G=(N, A)$, which consists of a finite set of nodes $N=\{1,2, \ldots, n\}$ and a set of directed $\operatorname{arcs} A=\{(i, j),(k, l), \ldots,(s, t)\}$, which joins pairs of nodes in $N$. It is graphically illustrated in Figure 1.

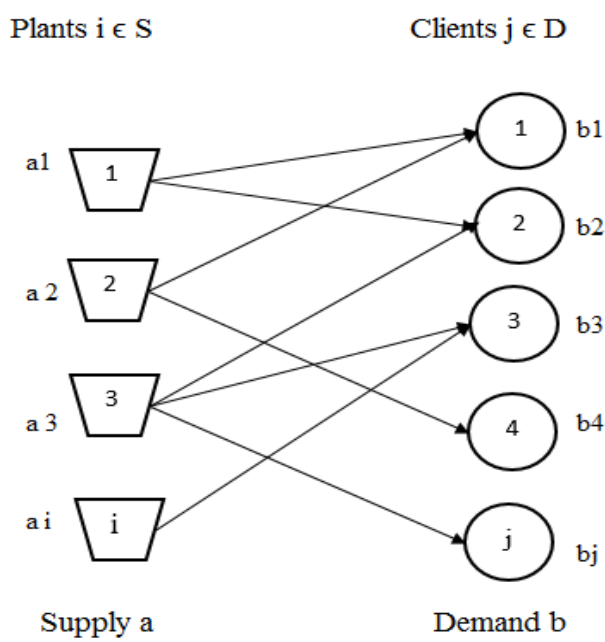

Figure 1. Transportation plan 


\subsection{Fixed-charge Logistic Model}

The FCTP is much more difficult to solve due to the presence of fixed costs, which cause discontinuities in the objective function. In the fixed-charge logistic model, two types of costs are considered simultaneously when the best course of action is selected: (1) variable costs proportional to the activity level; and (2) fixed costs. The FCTP seeks the determination of a minimum cost transportation plan for a homogeneous commodity from a number of plants to a number of customers. It requires the specification of the level of supply at each plant, the amount of demand at each customer, and the transportation cost and fixed cost from each plant to each customer. The goal is to allocate the supply available at each plant so as to optimize a criterion while satisfying the demand at each customer. The usual objective function is to minimize the total variable cost and fixed costs from the allocation. It is one of the simplest combinatorial problems involving constraints. The fixedcharge logistic model with $I$ plants and $J$ customers can be formulated as follows:

$$
\begin{aligned}
& \operatorname{Min} \mathrm{Z}=\sum_{i=1}^{m} \sum_{j=1}^{n}\left(C_{i j} x_{i j}+f_{i j} y_{i j}\right) \\
& \sum_{j=1}^{n} x_{\gamma}=S \quad i=1,2, \ldots ., m \\
& \sum_{i=1}^{m} x_{i j}=S \quad i=1,2, \ldots . n \\
& X_{i j} \geq 0 \quad i=1,2, \ldots, \text { mand } j=1,2, \ldots, n \\
& y_{i j}=0 \quad \text { if } \quad x_{i j}=0 \\
& y_{i j}=1 \quad \text { if } \quad x_{i j}>0
\end{aligned}
$$

$x_{i j}$ : Unknown quantity to be transported from source $i$ to destination $j$;

$c_{i j}$ : Variable transportation cost from source i to destination $j$

$f_{i j}$ : Fixed transportation cost associated with $\operatorname{road}(i, j)$;

$y_{i j}$ : A binary variable which is 1 if $x_{i j}>0$ and 0 if $x_{i j}=0$;

$S_{i}$ : Amount of supply at source $i$

$D_{j}$ : Amount of demand at destination $j$.

\section{PRIORITY-BASED GENETIC ALGORITHM FOR FIXED CHARGE LOGISTIC MODEL}

In order to apply the genetic algorithm $(\mathrm{GA})$, it is necessary to chose and adaptate the representation method for the solution of the problem. There are several methods of representation that are used to solve logistics models using GA, there is the matrix representation [15], representation by the number of prüfer [16], and there is also the priority based representation which is a new and more adapted for encoding and decoding the logistics models. It is first used by Gen and Altiparmak [17] to code and decode a two-stage transport problem. Then, the GA consists to several steps: Initialization processes (Generation of an population of solutions), evaluation, selection, crossover and mutation operators to create a new population of solutions.

\subsection{Encoding chromosome}

For the priority based representation, the solution (chromosome) is represented by an integral chain of length equaling the number of sources plus the number of clients. Each gene in this chromosome indicates the identification of a node (number).

In this representation, a gene in a chromosome contains two types of information:

a) The position of a gene to represent the nodes (source / destination)

b) The value of a gene which represents the priority of the node for the construction of a transport tree.

A chromosome consists of the priorities of sources and deposits to obtain a transport tree, its length is equal to the total number of sources $m$ and deposits $n$, ie. $(m+n)$. Each chromosome can be reconstructed in a random way and there is no need for a correction algorithm after the generation of a population [18].

\subsection{Selection and evaluation method}

Selection and evaluation are genetic processes to evaluate product solutions and compare them with existing chromosomes in order to choose the best to preserve them in memory. Therefore; the evaluation 
function will select or refuse an individual to retain only those individuals with the best cost according to the current population. In practice there are several types of selection applied in genetic algorithms, especially we employ the elitist mechanism; In fact, the best solution of the current population is selected and preserved in memory [19].
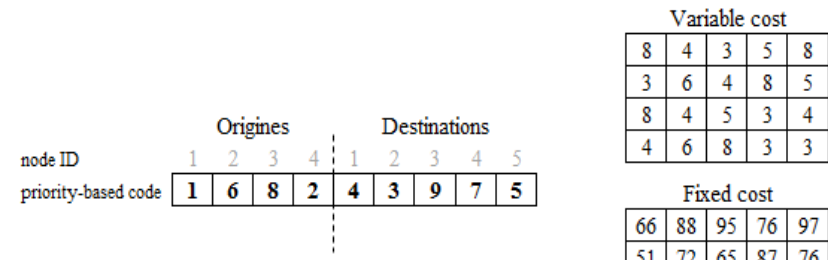

Fixed cost \begin{tabular}{|l|l|l|l|l|}
\hline 66 & 88 & 95 & 76 & 97 \\
\hline 51 & 72 & 65 & 87 & 76 \\
\hline
\end{tabular} \begin{tabular}{llll|l|l|}
\hline 51 & 72 & 65 & 87 & 76 \\
\hline
\end{tabular}

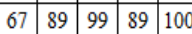

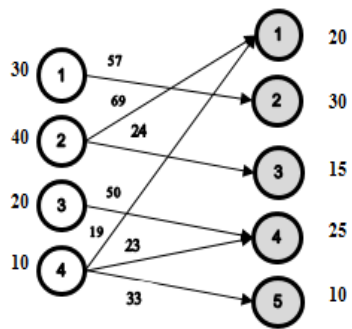
\begin{tabular}{|l|l|l|l|l|}
\hline 86 & 84 & 70 & 92 & 88 \\
\hline
\end{tabular}

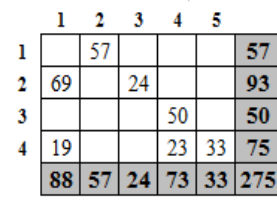

Figure 2. Priority-based chromosomes and transportation trees

\subsection{Adapted mutation}

Mutation operator operates by exchanging information within a chromosome. However, instead of using this operator between two parents, we use between two segments of a parent. We have chosen a swap mutation operator that is proposes by (Michalewicz, 1992) which permutes the values of two randomly selected positions for each chromosome to reduce the risk of reproducing a chromosome with the same solution [20], [21]. The procedure of swap mutation illustrated below works as follows:

\section{Procedure of the SWAP mutation}
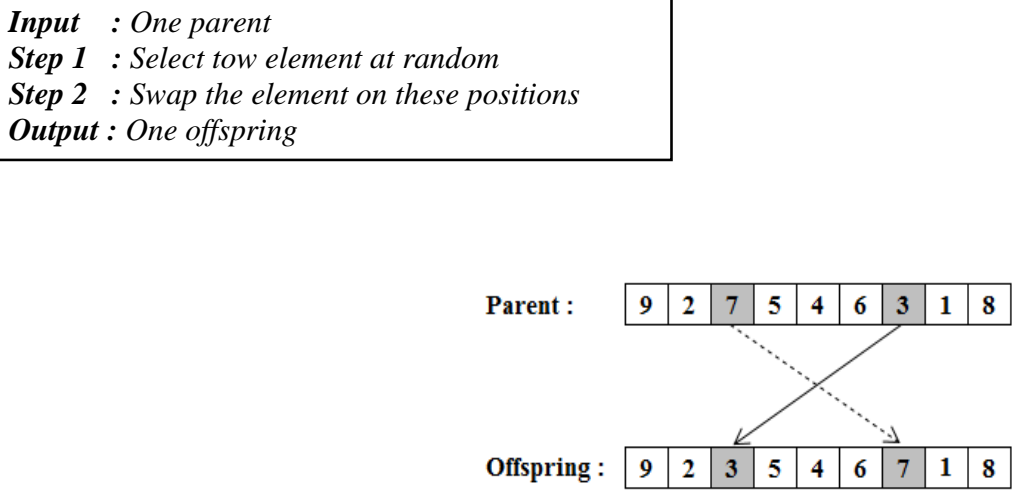

Figure 3. Example of the SWAP mutation operator

\subsection{Proposed crossover operator for FCTP}

The crossover operators are genetic processes. They work on two different parents (chromosomes) by combining the characteristics of the two chromosomes, they consist in applying procedures with a certain probability called the crossover rate $\left[P_{c} \in(0,1)\right]$ on the individuals selected to give birth to one or more (usually two) offspring. In this context, in order to ameliorate the performance of the GA to solve the fixed charge logistic model, we have chosen the two more peformante crossover operators (OX and PX) following a comparative study carried out for several ones [18]. Then, we proposed an hybridization of this two 
operators by highlighting the characteristics of each of them. The procedure of the proposed operator is presented bellow and the Figure 4. illustrate the procedure as an example.

\section{Procedure of the HOPX operator :}

Input: Tow parents;

Step $1:$ Select 1/3 substring from one parent at random;

Step $2:$ Select 1/4 substring and 1/4 of set of positions from same parent at random.

Step 3 : Produce a proto-child by copying the nodes on these positions into the corresponding positions of it;

Step 4 : Delete the nodes which are already selected from the second parent;

The resulted sequence of nodes contains the nodes of the proto-child needs;

Step 5 : Place the nodes into the unfixed positions of the proto-child from left to right according to the order of the sequence to produce one offspring;

Output: Tow offspring.

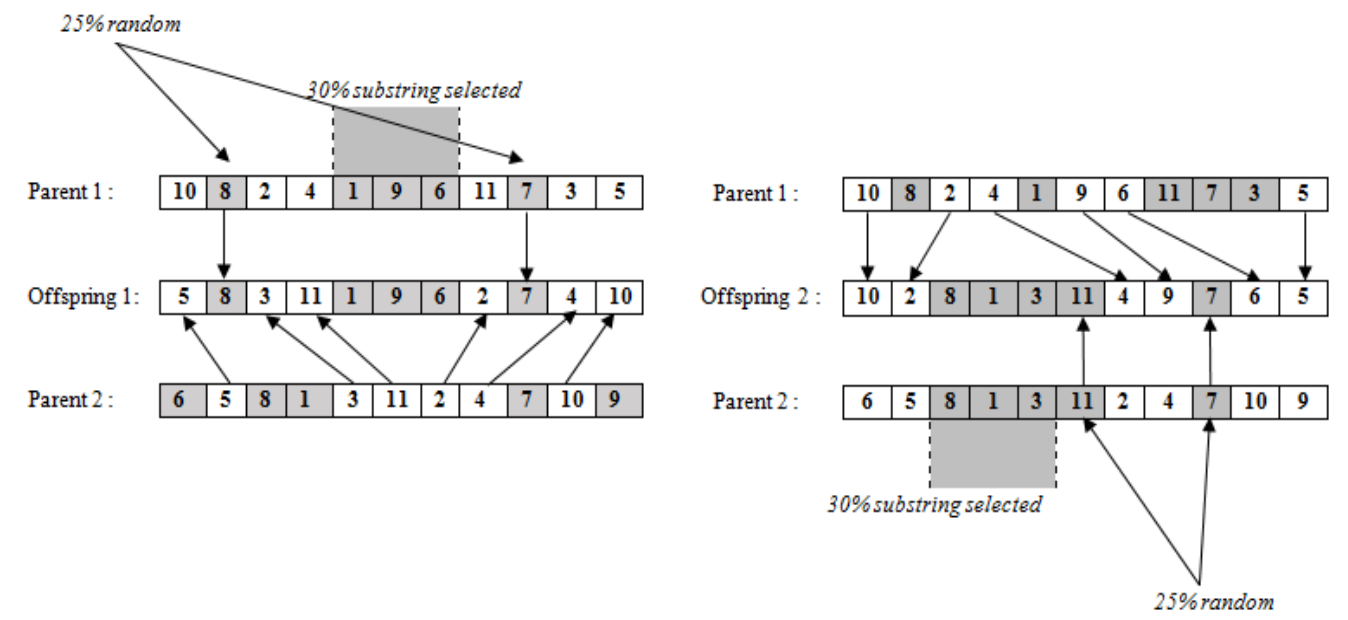

Figure 4. Example of the HOPX operator

\section{COMPUTATIONAL RESULTS}

The main objectives of this work are to improve the behavior of the genetic algorithm with respect to the crossover operators because they have an influence on the quality and performance of these algorithms and to establish to what extent the proposed algorithm solves the different types of logistic problems compared to the already used methods.

In this section, we have applied the developed HOPX operator to Logistic model with fixed cost in order to compare the results with those obtained using other adapted operators namely OPEX, OX, PX. In this study, we have chosen six instances 4x5, 5x10, 10x10, 10x20, 20x30, 30x50 already used in several articles [22], knowing that the optimal solution is known for small instances. Therefore, we have changed the genetic algorithm parameters, such as the number of iterations to see the influence of the latter on the results and on the quality of the solutions. This algorithm is coded in JAVA language in the NetBeans 8.0.2 IDE.

Table 1. presents the simulation results obtained from 20 times by the genetic algorithm based on each operator used to solve the logistic problem with fixed cost. For the results displayed in this table, we notice that the genetic algorithm with the HOPX operator allowed obtaining the optimal solution known for small instances as GAs with other crossover operators. However, for larger instances a better optimal solution is achieved with an improvement in computation time. Indeed, for small instances, $4 * 5$ and $5 * 10$, the obtained results show that GA with all crossover operators used allow to obtain the best chromosome satisfying the optimal solution. However, for problems with large size, with the proposed crossover operator HOPX, we are optimizing the objective function with a slight precision in terms of optimal solution. Then, the latter is more advantageous to the level of number of iterations with a reduced execution time. This means that HOPX is more appropriate than other operators that have already used to solve the fixed charge logistic model see Figure 5. and Figure 6. 
It should be noted that the genetic algorithm with the developed crossover operator is also applied to the linear logistic model which also shows its performance with respect to the GAs with the other mentioned operators.

Table 1. Best and average results by different operator for the test problems of Fixed-charge Logistic Model

\begin{tabular}{ccccccccccc}
\hline Problem & \multicolumn{2}{c}{ Parameter } & \multicolumn{2}{c}{ OPEX } & \multicolumn{2}{c}{ OX } & \multicolumn{2}{c}{ PX } & \multicolumn{2}{c}{ HOPX } \\
Size m x n & Popsize & Maxgen & Best & Avrg & Best & Avrg & Best & Avrg & Best & Avrg \\
\hline $4 \times 5$ & 20 & 300 & 9291 & 9291 & 9291 & 9291 & 9291 & 9291 & 9291 & 9291 \\
& 30 & 500 & 9291 & 9291 & 9291 & 9291 & 9291 & 9291 & 9291 & 9291 \\
$5 \times 10$ & 20 & 300 & 12718 & 12751 & 12718 & 12751 & 12718 & 12751 & 12718 & 12718 \\
& 30 & 500 & 12718 & 12734 & 12718 & 12734 & 12718 & 12734 & 12718 & 12718 \\
$10 \times 10$ & 20 & 500 & 13987 & 14074 & 13987 & 14139 & 14065 & 14133 & 13987 & 14047 \\
& 30 & 700 & 13934 & 14074 & 13987 & 14074 & 13934 & 14065 \\
$10 \times 20$ & 20 & 500 & 22258 & 22484 & 22376 & 22531 & 22198 & 22834 & 22150 & 22258 \\
& 30 & 700 & 22095 & 22484 & 22095 & 22198 & 22095 & 22532 & 22095 & 22198 \\
$20 \times 30$ & 20 & 500 & 32840 & 34119 & 33192 & 34581 & 32683 & 34414 & 32492 & 33142 \\
& 30 & 700 & 32526 & 33917 & 33917 & 33236 & 32492 & 33234 & 32471 & 32936 \\
$30 \times 50$ & 20 & 700 & 55611 & 56399 & 56399 & 56705 & 55450 & 56007 & 55269 & 55450 \\
& 30 & 1000 & 55143 & 55912 & 55912 & 55912 & 55106 & 55407 & 54114 & 55106 \\
\hline
\end{tabular}

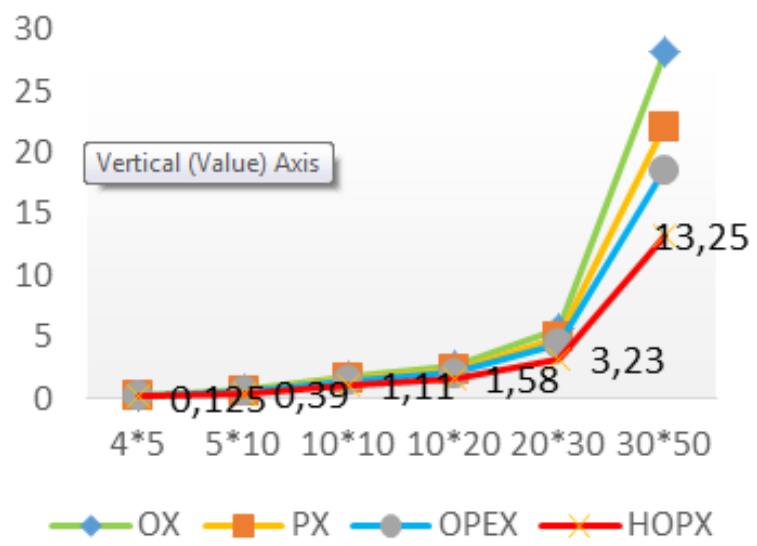

Figure 5. Average computation time for different operators for fixed charge Logistic Models

Table 2. gives the best chromosome for the different instances which represent the solution for the fixed charge logistic problem.

Table 2. Best chromosome for the test problems

\begin{tabular}{cl}
\hline Instance & \multicolumn{1}{c}{ Best chromosome } \\
\hline $4 \times 5$ & 168243975 \\
$5 \times 10$ & 111731314256124109158 \\
$10 \times 10$ & 8514121921615209107113164131817 \\
$10 \times 20$ & 245211091713191262912831820154711 \\
& 252326271630222814 \\
$20 \times 30$ & 5421331333023414892750112940162025 \\
& 322284332443862637492243671835148 \\
& 146341710154122145394719 \\
$30 \times 50$ & 595066478292517456356527528682521 \\
& 1940387653467142145561741226137236 \\
& 151133795744930437360524458264864 \\
& 2397520672470323623910455412177816 \\
& 16963771834378031 \\
\hline
\end{tabular}



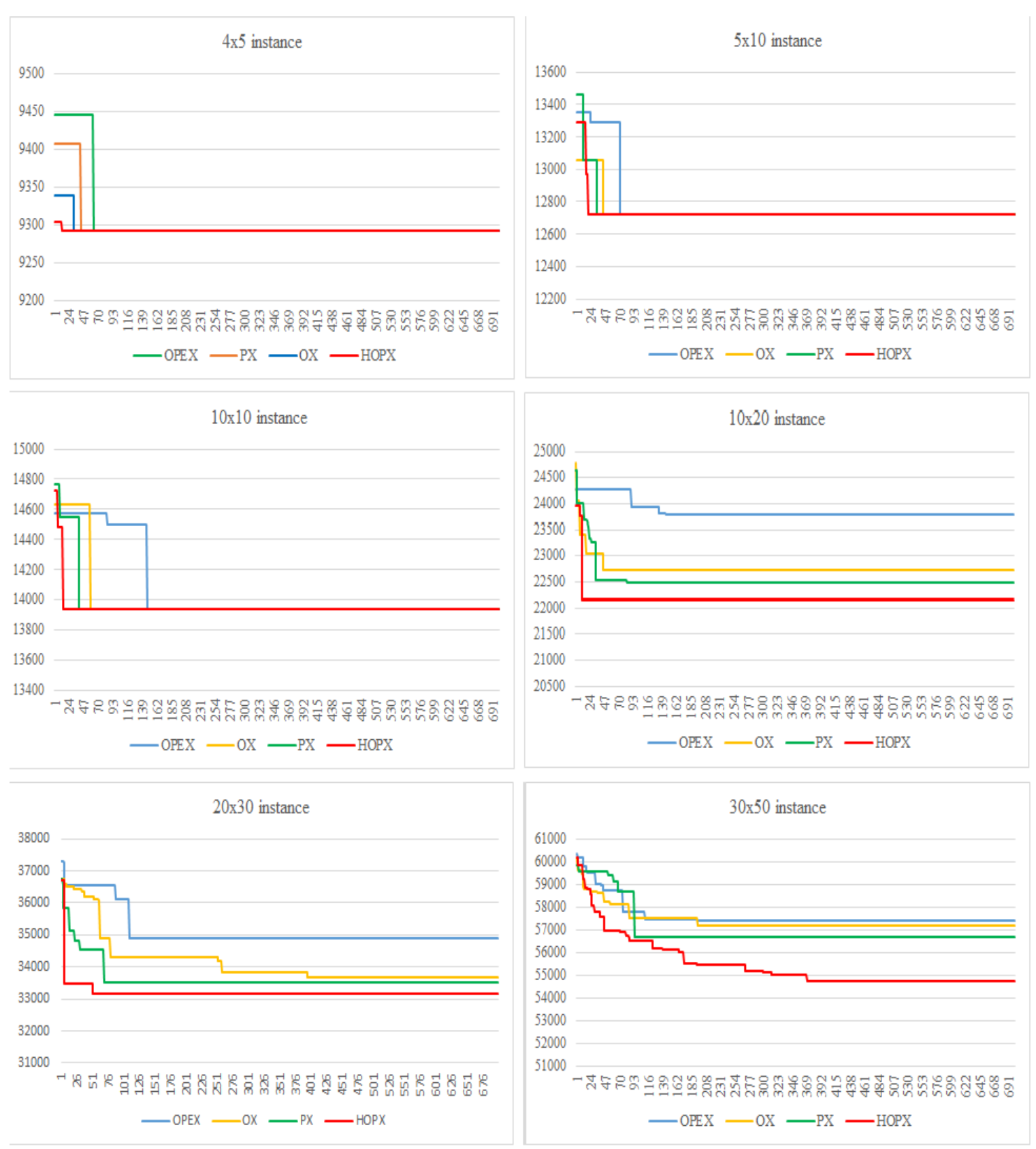

Figure 6. Optimum solution with different crossover operators OPEX, OX, PX, HOPX with different instances for fixed charge Logistic Models

\section{CONCLUSION}

In this work, we are interested in solving logistics models which are classified as NP-complet combinatorial problem called fixed charge transportation problem (FCTP). Its resolution by exact methods is very difficult, consequently the metaheuristics can be exploited such us genetic algorithms. Then, we are interested in improving the performance of GAs through the crossover operator that has a great effect. After presenting a comparison with three crossover operators (OX, OPEX, and PX) adapted to our problem with priority baseb encoding, we proposed a new hybrid operator with the two operators OX and PX that we called (HOPX). Numerical results was developed supporting the conclusion that the proposed HOPX operator is more efficient than the other presentedoperators either for the optimal solution level or the execution time, especially for larger instances. 


\section{REFERENCES}

[1] N. Balaji and N. Jawahar, "A simulated annealing algorithm for a two-stage fixed charge distribution problem of a supply chain", International Journal of Operational Research, pp. 192 - 215, 2010.

[2] F. L. Hitchcock, "The distribution of a product from several sources to numerous localities", Journal of Mathematical Physics, pp. 224 - 230, 1941.

[3] A. R. Fergusan and G. B. Dantzig, "The allocation of aircrafts to routes: An example of linear programming under uncertain demand". Management Science. vol 3, pp. 43-73, 1956.

[4] E. J. Russell, "Extension of Dantzig's Algorithm to Finding an Initial NearOptimal Basis for Transportation Problem", Operations Research, vol 17, pp. 187- 191, 1961.

[5] N. V. Reinfeld and W. R. Vogel, "Mathematical Programming", Prentice-Hall, Englewood Cliffs, N. 1958.

[6] W. M. Hirsch, G. B. Dantzig, "The fixed charge problem", Naval Research Logistics Quarterly, vol 15, pp. 413424, 1968.

[7] M. L. Balinski, "Fixed cost transportation problems", Naval Research Logistic Quarterly, vol. 8, (1961), pp. 41-54.

[8] V. Adlakha and K. Kowalski, "A simple heuristic for solving small fixed-charge transportation problems", Omega, vol. 31, pp. 205-211, 2003.

[9] Holland, J.,"Adaptation in natural and artificial systems". Ph.D thesis, University of Michigan Press, Ann Arbor, 1975 .

[10] Z. Zhu, D. Mulvaney and V. Chouliaras, "A Novel Genetic Algorithm Designed for Hardware Implementation", International journal of Computational Intelligence, vol 3, pp. 1842-1849, 2006.

[11] A. A. Hussein, "Improve The Performance of K-means by using Genetic Algorithm for Classification Heart Attack", International Journal of Electrical and Computer Engineering (IJECE), Vol. 8, No. 2, pp. 1256-1261, 2018.

[12] Q. Kotima, W. F. Mahmudy and V. N. Wijayaningrum, "Optimization of Fuzzy Tsukamoto Membership Function using Genetic Algorithm to Determine the River Water", International Journal of Electrical and Computer Engineering (IJECE), Vol. 7, No. 5, pp. 2838 - 2846, 2017.

[13] D. N. Le, "GA and ACO Algorithms Applied to Optimizing Location of Controllers in Wireless Networks", International Journal of Electrical and Computer Engineering (IJECE), Vol. 3, No. 2, pp. 221-229, 2013.

[14] K.A. De Jong and W. M. Spears, "Using Genetic Algorithms to Solve NP Complete Problems", Proceedings of the Third International Conference on Genetic Algorithms, 1989, pp. 124-132.

[15] G.A. Vignaux and Z. Michalewicz, "A genetic algorithm for the linear transportation problem", IEEE Transactions on Systems, Man, and Cybernetics, vol 21, 1991, pp. 445-452.

[16] M. Gen, R. Cheng, "Genetic Algorithms and Engineering Optimization", John Wiley and Sons, New York 2000.

[17] M. Gen, F. Altiparmak, L. Lin, "A genetic algorithm for two-stage transportation problem using priority-based encoding", OR Spectrum, vol 28, 2006, pp. 337-354.

[18] A. L. El Idrissi, C. Tajani and M. Sabbane, "New crossover operator for genetic algorithm to resolve the fixed charge transportation problem", Journal of Theoretical \& Applied Information Technology, Vol. 95 N. 8, pp. 16071617.

[19] B. Chakraborty and P. Chaudhuri, "On The Use of Genetic Algorithm with Elitism in Robust and Non parametric Multivariate Analysis", Austrian journal of statistics,, vol. 32( 1-2), 2003, pp 13-27.

[20] Z. Michalewicz, G.A. Vignaux and M. Hobbs, "A non-standard genetic algorithm for the nonlinear transportation problem", ORSA Journal on Computing, vol. 3, (1991), pp. 307-316.

[21] J. W. Stroup, "Allocation of launch vehicles to space missions: a fixed-cost transportation problem", Operations Research, vol. 15, No. 6, 1967, pp. 11-57.

[22] M. Lotfia, R. Tavakkoli, "A genetic algorithm using priority-based encoding with new operators for fixed charge transportation problems", Applied Soft Computing, vol. 13, 2013, pp. 2711-2726. 